\title{
Improving System Sensitivity via Surplus Controller Capacity
}

\section{by WILLIAM A. PORTER}

\section{Systems Engineering Laboratory, Department of Electrical Engineering}

University of Michigan, Ann Arbor, Michigan

ABSTRACT: In this paper a linear plant with variable characteristics is considered. It is assumed that the plant is driven optimally by an open-loop controller. For a particular task and a nominal plant characteristic the controller is assumed to have a surplus capacity (in the optimal sense). This surplus capacity is used to minimize the effects of fuctuations in the plant characteristics about the nominal.

Three criteria are formulated for optimally using the controller surplus capacity. Solutions to the three cases are derived and compared. The analysis provides insight into problems of optimal control containing a parameter.

\section{Introduction}

The problem of system sensitivity takes many forms depending on the disturbances considered and the design constraints in effect. Several authors: Pagurek (1), Witsenhausen (2), Holtzman and Horing (3) and Porter (4) consider sensitivity problems in optimal systems (or systems under optimal control). Rohrer and Sobral (5), and Porter (6) consider the problem of defining a sensitivity measure. Perkins and Cruz (7), and Porter (8) investigate the reduction of sensitivity in multivariate systems.

This paper is concerned with a mixture of sensitivity and optimality. We consider specifically linear systems under minimum energy control. The particular problems discussed here do not appear to have been considered in any of the earlier analyses.

Assume that for a nominal plant the controller capacity exceeds the control requirements. The investigation is concerned with the question: How can the surplus capacity be used to minimize the effects of fluctuations in the plant characteristics?

It is well known that minimum energy problems in linear systems have a natural identification in terms of linear transformations between appropriate Hilbert spaces. Throughout the present analysis, $H_{1}$ and $H_{2}$ denotes Hilbert spaces and the bounded linear transformation $T: H_{1} \rightarrow H_{2}$ represents the nominal system map. We denote the system disturbance by the symbol $\delta T$, which is also bounded and linear.

Let $\xi \in \mathrm{H}_{2}$ represent the system task. Assume that $\xi$ has a preimage under $T$ with less than the available energy. How can the surplus energy be used to minimize the effects of $\delta T$ ? 
The problem just posed may be givon several diverse analytic interpretations. Attention is concentrated on two specific cases which reflect different approaches to the basic problem. The first and most direct of the two cases is as follows:

\section{Problem I.}

For fixed $\xi \in H_{2}$ such that $\left\|T^{\dagger} \xi\right\|<k$, find $u \in T^{-1}(\xi)$ which satisfied $\|u\| \leq k$ while minimizing $\|\delta T u\|$.

In the statement of this problem, $T^{-1}(\xi)$ denotes the set of all preimages of $\xi$ under $T$ and $T^{\dagger}$ denotes the pseudo inverse of $T$. First we note that if $u_{\xi}=T^{\dagger} \xi$ happens to be in the null space of $\delta T$ this control is a solution to the problem. We assume that this uninteresting case does not occur. Secondly, if $u \in H_{1}$ is a solution, then it may be decomposed in the form

$$
u=v+u_{\xi}, \quad v \in N(T), \quad u_{\xi}=T^{\dagger} \xi \in N(T)^{\perp}
$$

where $N(T)$ is the null space of $T$ and $N(T)^{\perp}$ its orthogonal complement. This decomposition follows easily from the fact that $N(T)$ is closed and that $T^{\dagger} \xi$ is the unique preimage of $\xi$ in $N(T)^{\perp}$. Since

$$
\|u\|^{2}=\|v\|^{2}+\|u\|^{2}
$$

it follows that an equivalent statement of Problem I is: Find an element $v \in N(T)$ which minimizes $\left\|\delta T u_{\xi}+\delta T v\right\|$ while satisfying $\|v\|^{2} \leq k^{2}-\left\|u_{\xi}\right\|^{2}$.

Let $P$ denote the orthogonal projection of $H_{1}$ onto $N(T)$. Then if $v$ is a solution of Problem I, clearly

$$
\delta T P v=\delta T v .
$$

Moreover, if $\omega$ is any element satisfying $\|\omega\|^{2} \leq k^{2}-\left\|u_{\xi}\right\|^{2}$ and minimizing $\left\|\delta T u_{\xi}+\delta T P \omega\right\|$ then $v=P \omega$ is a solution to Problem I. Thus by introducing the transformation $V=\delta T P$ the constraint $v \in N(T)$ will automatically be satisfied.

Now let $\overline{R(V)}$ denote the closure of the range of $V$. If $\xi^{\prime}$ is the orthogonal projection of $\xi$ on $\overline{R(V)}$ and if $\xi^{\prime} \in R(V)$ then $V^{-1}\left(\xi^{\prime}\right)$ is nonempty. If the minimum element of this set has norm $\leq k^{2}-\left\|u_{\xi}\right\|^{2}$ than a solution to Problem I is obtained by choosing $v$ to be this minimum element. If the norm of this minimum element exceeds $k^{2}-\left\|u_{\xi}\right\|^{2}$ or if $\xi^{\prime} \notin R(V)$ than any solution to the problem must have $\|v\|^{2}=k^{2}-\left\|u_{\xi}\right\|^{2}$. It is this last case which merits further study.

If $\delta \xi=\delta T u_{\xi}=\delta T T^{\dagger} \xi$ and $\epsilon$ is a scalar such that $k^{2}=(1+\epsilon)\left\|T^{\dagger} \xi\right\|^{2}$ then it suffices to minimize the functional

$$
\|\delta \xi+V v\|
$$

subject to the constraint $\|v\|^{2}=\epsilon\left\|T^{\dagger} \xi\right\|^{2}$. The Lagrange multiplier technique 
is used. Forming the functional

$$
J(v)=\|\delta \xi+V v\|^{2}+\lambda\left(\|v\|^{2}-\epsilon\left\|T^{\dagger} \xi\right\|^{2}\right)
$$

it is easily shown that

$$
J(v+\delta v)-J(v)=2\left\langle\mathrm{~V}^{*} \delta \xi+\mathrm{V}^{*} V v+\lambda v, \delta v\right\rangle .
$$

Hence, at the optimal $v$, the equation

$$
V^{*} \delta \xi+V^{*} V^{\gamma} v+\lambda v=0
$$

must hold. The optimal control must obviously satisfy

$$
v=-\left(\lambda I+V^{*} V\right)^{-1} V^{*} \delta \xi, \quad \lambda>0
$$

where the scalar $\lambda$ has yet to be determined.

From Eq. 1 it follows that

$$
\|v\|^{2}=\left\langle\delta \xi, V\left(\lambda I+V^{*} V\right)^{-2} V^{*} \delta \xi\right\rangle=\left\langle\delta \xi,\left(\lambda I+V V^{*}\right)^{-2} V V^{*} \delta \xi\right\rangle
$$

and hence that $\lambda$ must satisfy the condition

$$
\left\langle\delta \xi,\left(\lambda I+V V^{*}\right)^{-2} V V^{*} \delta \xi\right\rangle=\epsilon\left\|T^{\dagger} \xi\right\|^{2}
$$

For $\delta \xi \in R(V)^{\perp}$ it is well-known that $V^{*} \delta \xi=0$; hence Eq. 2 can be satisfied only if $\epsilon=0$. This is the case where the control $v=0$ is optimal for our problem. Moreover, it is not difficult to show that $\overline{R(V)}$ and $R(V)^{\perp}$ are reducing subspaces for $\left(\lambda I+V V^{*}\right)^{-2}$; hence, only the component of $\delta \xi$ in $\overline{R(V)}$ effects the right hand side of Eq. 2. Rather than taking projections we assume that $\delta \xi \in \overline{R(V)}$.

To show that Eq. 2 can be satisfied consider first the case of very small $\lambda$. Formally set $\lambda=0$ and interpret $\left(V V^{*}\right)^{-1}$ as an operator densely defined in $\overline{R(V)}$. Now if $\delta \xi \in R(V)$, then $\delta \xi=V \varphi$ for some $\varphi$ and we choose the $\varphi$ of minimum norm which satisfies this equation. Than (since $\varphi=V^{*}\left(V V^{*}\right)^{-1} \delta \xi$ ) the equality

$$
\left\langle\delta \xi,\left(V V^{*}\right)^{-2}\left(V V^{*}\right) \delta \xi\right\rangle=\left\langle\varphi, V^{*}\left(V V^{*}\right)^{-1} V \varphi\right\rangle=\|\varphi\|^{2}
$$

holds. However, the existence of a $\varphi$ such that $\|\varphi\| \leq \epsilon\left\|^{\dagger} \xi\right\|^{2}$ and $\delta \xi=V \varphi$ is one of the special cases considered earlier and hence for small $\lambda$ the left hand side of Eq. 2 exceeds the right hand side.

Now it can be shown that the left hand side of Eq. 2 is monotonic decreasing with increasing $\lambda$ and approaches zero as $\lambda \rightarrow \infty$. Thus, Eq. 2 is satisfied for a unique $\lambda$. To illustrate, assume that $V^{*} V$ is compact with the spectral expansion

$$
V^{*} V=\sum_{i=1}^{\infty} e_{i}>\mu_{i}<e_{i}
$$


in terms of the orthonormal basis $\left\{e_{i}\right\}$ for $H_{2}$. Then if $\left\{\varphi_{i}\right\}$ are the Fourier coefficients of $\varphi$ along this basis where $\delta \xi=V \varphi$, we obtain

$$
\left\langle V^{*} \delta \xi,\left(\lambda I+V^{*} V\right)^{-2} V^{*} \delta \xi\right\rangle=\sum_{i=1}^{m} \frac{\left(\mu_{i} \varphi_{i}\right)^{2}}{\left(\lambda+\mu_{i}\right)^{2}}
$$

which clearly exhibits the monotonic property. Thus Eqs. 1 and 2 determine the solution to Problem I under the assumption that $\delta \xi \in R(V)$ and $\left\|V^{\dagger} \delta \xi\right\| \geq$ $\epsilon\left\|T^{\dagger} \xi\right\|$.

In the next two problems the functional $J$ defined by

$$
J(u)=\|u\|^{2}+\alpha\|\delta T u\|^{2}
$$

plays an important role. Here the energy of a control and the system output disturbance corresponding to that control are weighted together to form the functional.

\section{Problem II.}

Assume for $\xi \in H_{2}$ that $\left\|T^{\dagger} \xi\right\|<k$. Find the largest $\alpha$ for which a control $u$ of norm $k$ exists such that $u$ minimizes $J$ while satisfying $\xi=T u$.

In other words, we wish to determine the maximum weight that can be given to the output disturbance such that a control exists, which is optimal with respect to $J$ and the constraint, while having acceptable energy. In analyzing this problem advantage is taken of the fact that the bilinear functional

$$
(x, y)=\left\langle x,\left(I+\alpha \delta T^{*} \delta T\right) y\right\rangle \quad x, y \in H_{1}
$$

is an inner product on $H_{1}$. Moreover, the norm induced by this inner product is precisely the functional $J$ (this new norm is actually equivalent to the given norm). Thus, if $T$ \# denotes the pseudo inverse of $T$ with respect to the norm $J$, Problem II may be rephrased as finding the largest $\alpha$ for which $\|T \# \xi\|=k$.

For convenience the positive self adjoint operator $K=\delta T^{*} \delta T$ is introduced. Then it can be shown (see (9), Chap. IV) that

$$
T^{\#}=(I+\alpha K)^{-1} T^{*}\left[T(I+\alpha K)^{-1} T^{*}\right]^{-1}
$$

where $T^{*}$ and $\delta T^{*}$ still denote adjoints with respect to the original norm. (Since $T^{\star}$, the adjoint of $T$ with respect to the new norm, satisfies $T^{\star}=(I+\alpha K)^{-1} T^{*}$ the familiar identification $T^{\#}=T^{\star}\left(T T^{\star}\right)^{-1}$ holds as usual.)

In the following lemma, $T$ is onto and $P$ denotes the orthogonal projection of $H$ onto $N(T)$.

Lemma I. Whenever the indicated inverse exists

$$
T^{\#}=(I+\alpha P K)^{-1} T^{\dagger}
$$

holds. 
William A. Porter

Proof: Consider first the identity chain

$$
\begin{aligned}
T^{\prime}(I+\alpha K)^{-1} T^{*} & =T(I+\alpha K)^{-1} T^{*} \\
& =T\left[(I+\alpha K)^{-1}(I+\alpha K-\alpha K)\right] T^{*} \\
& =T\left[I-\alpha(I+\alpha K)^{-1} K\right] T^{*} \\
& =T T^{*}-\alpha T(1+\alpha K)^{-1} K T^{*} .
\end{aligned}
$$

Since $T$ is onto $T T^{*}$ has a bounded inverse and $T^{*}$ is one to one on all of $H$. Using these facts with the above equality establishes the identity

$$
\begin{aligned}
T^{*}\left(T T^{*}\right)^{-1}\left[T(I+\alpha K)^{-1} T^{*}\right] & =T^{*}\left[I-\alpha\left(T T^{*}\right)^{-1} T(I+\alpha K)^{-1} K T^{*}\right] \\
& =T^{*}-\alpha T^{*}\left(T T^{*}\right)^{-1} T(I+\alpha K)^{-1} K T^{*}
\end{aligned}
$$

Recognizing now that $T^{\dagger}=T^{*}\left(T T^{*}\right)^{-1}$ and $(I-P)=T^{*}\left(T T^{*}\right)^{-1} T$ this becomes

$$
\begin{aligned}
T_{\dagger}^{\dagger}\left[T(I+\alpha K)^{-1} T^{*}\right] & =T^{*}-\alpha(I-P)(I+\alpha K)^{-1} K T^{*} \\
& =\left[I-\alpha(I-P)(I+\alpha K)^{-1} K\right] T^{*} \\
& =\left[I-\alpha(I-P) K(I+\alpha K)^{-1}\right] T^{*} \\
& =[(I+\alpha K)-\alpha(I-P) K](I+\alpha K)^{-1} T^{*} \\
& =(I+\alpha P K)(I+\alpha K)^{-1} T^{*}
\end{aligned}
$$

which by using inverses establishes the lemma.

Before proceeding with the solution to Problem II, a related problem is considered.

\section{Problem III:}

In Problem II set $k^{2}=(1+\epsilon)\left\|T^{\dagger} \xi\right\|^{2}$ and determine $\alpha$ from the equality

$$
J\left(T^{\# \xi}\right)=(1+\epsilon)\left\|T^{\dagger} \xi\right\|^{2} .
$$

Problem III evidently reduces to a study of the scalar equation

$$
\left\|T^{\# \xi}\right\|^{2}+\alpha\left\|\delta T T^{\# \xi}\right\|^{2}=(1+\epsilon)\left\|T^{\dagger} \xi\right\|^{2} .
$$

Recalling that $\delta T^{*} \delta T=K$ this equation becomes

$$
\left\langle T^{*} \xi,(I+\alpha K) T^{\#} \xi\right\rangle=(1+\epsilon)\left\langle T^{\dagger} \xi, T^{\dagger} \xi\right\rangle .
$$

Using the above lemma we have

$$
\left\langle T^{\dagger} \xi,\left[(I+\alpha K P)^{-1}(I+\alpha K)(I+\alpha P K)^{-1}-(1+\epsilon) I\right] T^{\dagger} \xi\right\rangle=0
$$

which may be rewritten as

$$
\left\langle\xi,\left(T^{\dagger}\right)^{*} W(\alpha) T^{\dagger} \xi\right\rangle-\epsilon\left\langle\xi,\left(T^{\dagger}\right)^{*} T^{\dagger} \xi\right\rangle=0
$$


where

$$
W(\alpha)=(I+\alpha K P)^{-1}(I+\alpha K)(I+\alpha P K)^{-1}-I
$$

Thus a Corollary of Lemma I follows:

Corollary. The dependence between $\alpha$ and $\epsilon$ is determined by

$$
\left\langle\xi,\left(T^{\dagger}\right)^{*} W(\alpha) T^{\dagger} \xi\right\rangle=\epsilon\left\langle\xi,\left(T^{\dagger}\right)^{*} T^{\dagger} \xi\right\rangle .
$$

Lemma II. Whenever $I+\alpha P K$ has a bounded inverse:

(a) $I+\alpha K P$ has a bounded inverse.

(b) $I-(I+\alpha K P)^{-1}=\alpha K P(I+\alpha K P)^{-1}$.

(c) $(I+\alpha K P)^{-1}(I+\alpha P K)^{-1}-(I+\alpha P K)^{-1}-\left(I+\alpha K I^{\prime}\right)^{-1}+I=$ $\alpha^{2} K P K(I+\alpha P K)^{-2}$.

Proof: Part (a) follows easily from the identity

$$
(I+\alpha K P)^{-1}=I-\alpha K(I+\alpha P K)^{-1} P
$$

where first the right hand side defines the left; then it is shown that the left hand side is the inverse of $I+\alpha K P$. Similarily part (b) follows by clearing fractions. To prove (c), first rewrite the left hand side of this identity to obtain

$$
\left[I-(I+\alpha K P)^{-1}\right]\left[I-(I+\alpha P K)^{-1}\right]=\alpha^{2} K P K(I+\alpha P K)^{-2}
$$

Then by using part (b) this becomes

$$
\alpha K P(I+\alpha K P)^{-1} \alpha P K(I+\alpha P K)^{-1}=\alpha^{2} K P K(I+\alpha P K)^{-2}
$$

which is equivalent to

$$
(I+\alpha K P)^{-1} K P K=K P K(I+\alpha P K)^{-1} .
$$

This last equality is easily verified and completes the proof.

\section{Corollary.}

$$
\left(T^{\dagger}\right)^{*} W(\alpha) T^{\dagger}=\alpha\left(T^{\dagger}\right)^{*}\left[K(I+\alpha P K)^{-1}\right] T^{\dagger}
$$

Proof: Using the definition (Eq. 6) of $W(\alpha)$ and some elementary operations, the equality chain

$$
\begin{aligned}
W(\alpha)= & (I+\alpha K P)^{-1}(I+\alpha K)(I+\alpha P K)^{-1}-I \\
= & (I+\alpha K P)^{-1}(I+\alpha P K)^{-1}-(I+\alpha K P)^{-1}-(I+\alpha P K)^{-1}+I \\
& \quad+(I+\alpha K P)^{-1}+(I+\alpha P K)^{-1}+\alpha K(I+\alpha P K)^{-2}-2 I
\end{aligned}
$$


is established. Using part (c) of the lemma, we obtain

$$
\begin{aligned}
W(\alpha)= & \alpha^{2} K P K(I+\alpha P K)^{-2}+\alpha K(I+\alpha P K)^{-2}+(I+\alpha K P)^{-1} \\
& +(I+\alpha P K)^{-1}-2 I \\
= & \alpha K(I+\alpha P K)(I+\alpha P K)^{-2}+(I+\alpha K P)^{-1}+(I+\alpha P K)^{-1}-2 I \\
= & \alpha K(I+\alpha P K)^{-1}+(I+\alpha K P)^{-1}+(I+\alpha P K)^{-1}-2 I .
\end{aligned}
$$

The corollary now follows from this equation and the fact that

$$
(I+\alpha K P)^{-1} T^{\dagger}=T^{\dagger} .
$$

To verify this latter assertion recall that $P$ projects on $N(T)$ while the range of $T^{\dagger}$ is in $N(T) \perp$. Therefore $P T^{\dagger}=0$ and hence

$$
T^{\dagger}=(I+\alpha K P)^{1}(I+\alpha K P) T^{\dagger}=(I+\alpha K P)^{-1} T^{\dagger}
$$

Since $K$ and $P$ are self-adjoint this equality implies also that

$$
\left(T^{\dagger}\right)^{*}=\left(T^{\dagger}\right)^{*}(I+\alpha P K)^{-1}
$$

and hence

$$
\left(T^{\dagger}\right)^{*}\left[(I+\alpha K P)^{-1}+(I+\alpha P K)^{-1}-2 I\right] T^{\dagger}=0
$$

which completes the proof of the corollary. An immediate consequence is that in Problem III the dependence of $\alpha$ on $\epsilon$ is expressed by

$$
\alpha\left\langle T^{\dagger} \xi, K(I+\alpha P K)^{-1} T^{\dagger} \xi\right\rangle=\epsilon\left\|T^{\dagger} \xi\right\|^{2} .
$$

Consider now Problem II with $k^{2}=(1+\epsilon)\left\|T^{\dagger} \xi\right\|^{*}$. Evidently it suffices to study the equation

$$
\left\langle T^{\dagger} \xi,(I+\alpha K P)^{-1}(I+\alpha P K)^{-1} T^{\dagger} \xi\right\rangle=(1+\epsilon)\left\langle T^{\dagger} \xi, T^{\dagger} \xi\right\rangle .
$$

Since

$$
(I+\alpha K P)^{-1}(I+\alpha P K)^{-1}-I=W(\alpha)-\alpha K(I+\alpha P K)^{-2}
$$

it follows from the corollary to Lemma II that

$$
\begin{aligned}
\left\langle T^{\dagger} \xi,\left[(I+\alpha K P)^{-1}(I+\right.\right. & \left.\left.\alpha P K)^{-1}-I\right] T^{\dagger} \xi\right\rangle \\
& =\left\langle T^{\dagger} \xi,\left[\alpha K(I+\alpha P K)^{-1}-\alpha K(I+\alpha P K)^{-2}\right] T^{\# \xi}\right\rangle \\
& =\alpha^{2}\left\langle T^{\dagger} \xi, K P K(I+\alpha P K)^{-2} T^{\dagger} \xi\right\rangle .
\end{aligned}
$$


Thus in Problem II the dependence of $\alpha$ on $\epsilon$ is determined by

$$
\alpha^{2}\left\langle T^{\dagger} \xi, K P K(I+\alpha P K)^{-2} T^{\dagger} \xi\right\rangle=\epsilon\left\|T^{\dagger} \xi\right\|^{2} .
$$

\section{Comparison of the Solutions}

In comparing the solutions of the preceding three problems it is helpful to rework the results of Problem I. Recall first that $\delta \xi=\delta T u_{\xi}=\delta T T^{\dagger} \xi$ and that $V=\delta T P$. Thus it follows that

$$
V^{*} V=P \delta T^{*} \delta T P=P K P
$$

and that Eq. 1 may be rewritten as

$$
\begin{aligned}
v & =-(\lambda I+P K P)^{-1}\left(P \delta T^{*}\right)\left(\delta T^{\dagger} \xi\right) \\
& =-(\lambda I+P K P)^{-1} P K T^{\dagger} \xi \\
& =-P K(\lambda I+P K)^{-1} T^{\dagger} \xi .
\end{aligned}
$$

Let $T^{\ddagger}$ denote the mapping of $\xi$ (for every $\xi$ ) to the solution of Problem I. Since $T^{\ddagger} \xi=T^{\dagger} \xi+v$ it follows that

$$
T^{\ddagger}=\left[I-P K(\lambda I+P K)^{-1}\right] T^{\dagger} .
$$

If $\lambda=1 / \beta$ observe that $(\lambda I+P K)^{-1}=\beta(I+\beta P K)^{-1}$ and that the identity

$$
(I+\beta P K)^{-1}=I-\beta P K(I+\beta P K)^{-1}
$$

holds. Thus $T^{\ddagger}$ may be identified as in the equality

$$
T^{\ddagger}=(I+\beta P K)^{-1} T^{\dagger} .
$$

Consider now Eq. 2. By direct substitution and some elementary rearrangements the equality chain

$$
\begin{aligned}
\left\langle\delta \xi, V\left(I+V^{*} V\right)^{-2} V^{*} \delta \xi\right\rangle & =\left\langle T^{\dagger} \xi, \delta T^{*} \delta T P(I+P K P)^{-2} P \delta T^{*} \delta T T^{\dagger} \xi\right\rangle \\
& =\beta^{2}\left\langle T^{\dagger} \xi, K P K(I+\beta P K)^{-2} T^{\dagger} \xi\right\rangle .
\end{aligned}
$$

It therefore follows that $\beta$ must satisfy the equality

$$
\beta^{2}\left\langle T^{\dagger} \xi, K P K(I+\beta P K)^{-2} T^{\dagger} \xi\right\rangle=\epsilon\left\|T^{\dagger} \xi\right\|^{2} .
$$

In view of Eqs. 3 and 10 it is obvious that both $T^{\#}$ and $T^{\ddagger}$ have the form $(I+\gamma P K)^{-1} T^{\dagger}$. In view of Eqs. 9 and 11 it is obvious that the solutions to Problems I and II are identical. The form of the optimal control in Problem III is the same as in Problems I and II. The value of $\gamma$, however, is different being determined by Eq. 8 . 
It is also of interest to compute the norm of $E_{\xi}$ which is the actual miss distance. Recalling that $P$ has range in $N(T)$ it follows that

$$
T(I+\gamma P K)^{-1}=T
$$

holds for all $\gamma$. This, together with the identity $T T^{\dagger} \xi=\xi$, establishes the equality chain

$$
\begin{aligned}
E_{\xi} & =\xi-(T+\delta T)(I+\gamma P K)^{-1} T^{\dagger} \xi \\
& =\delta T(I+\gamma P K)^{-1} T^{\dagger} \xi .
\end{aligned}
$$

Hence, we obtain

$$
\begin{aligned}
\left\|E_{\xi}\right\|^{2} & =\left\|\delta T(I+\gamma P K)^{-1} T^{\dagger} \xi\right\|^{2} \\
& =\left\langle T^{\dagger} \xi, K(I+\gamma P K)^{-2} T^{\dagger} \xi\right\rangle .
\end{aligned}
$$

Thus, in all three problems the system errors have the same form differing only in the value of the scalar $\gamma$.

Since the distinction of the three problems hinges on the two scalars involved, a comparison of Eqs. 8 and 11 is of interest. Since $K=\delta T^{*} \delta T$ Eqs. 8 and 11 may be rewritten as

$$
\begin{aligned}
& f_{2}(\alpha)=\alpha\left\langle\eta,(I+\alpha Q)^{-1} \eta\right\rangle=\epsilon\left\|T^{\dagger} \xi\right\|^{2} \\
& f_{1}(\beta)=\beta^{2}\left\langle\eta, Q(I+\beta Q)^{-2} \eta\right\rangle=\epsilon\left\|T^{\dagger} \xi\right\|^{2}
\end{aligned}
$$

respectively, where $\eta=\delta \xi=\delta T T^{\dagger} \xi$ and $Q=\delta T P \delta T^{*}$. In the Appendix it is shown that both $f_{1}$ and $f_{2}$ are monotone increasing (or monotone nondecreasing) functions on $[0, \infty)$. Further, it is shown that $f_{1}(\gamma) \geq f_{2}(\gamma)$ holds for $\gamma \in[0, \infty)$. Consequently, if $\alpha, \beta$ are solutions to the equations

$$
f_{2}(\alpha)=\epsilon\left\|T^{\dagger} \xi\right\|^{2}=f_{1}(\beta)
$$

for any $\epsilon \geq 0$ then $\alpha \leq \beta$. The Appendix also shows that

$$
\left\|E_{\xi}\right\|^{2}=\left\langle\eta,(I+\gamma Q)^{-2} \eta\right\rangle=f_{4}(\alpha)
$$

is a monotone decreasing function with respect to $\gamma$. Therefore, the common solution to Problems I and II is always better than or at least equal to the solution of Problems III as far as system errors are concerned.

\section{Example I.}

We examine two degenerate cases. First consider the case where the system disturbance takes the form of a pure gain constant change. That is, $\delta T=\mu T$ for some scalar $\mu \neq 0$. Then $K=\mu^{2} T^{*} T$ and since $P$ annihilates $T^{*}$ it follows that $P K=0$ and hence $T^{\ddagger}=T^{*}=T^{\dagger}$. This result verifies the obvious fact that the constraint $T u=\xi$ fixes $\delta \xi=\delta T u=\mu T u=\mu \xi$; hence, no minimizing of sensitivity is possible. More generally, if $\delta T$ is a distortion $(6,10)$ then $P K=0$ and the same conclusion holds. 
As a second specific case assume that $P$ and $K$ commute. Then

$$
T^{\#}=T^{\ddagger}=(I+\gamma P K)^{-1} T^{\dagger}=(I+\gamma K P)^{-1} T^{\dagger}=T^{\dagger}
$$

holds for any $\gamma$. Also

$$
\left\|E_{\xi}\right\|^{2}=\left\|\delta T(I+\gamma P K)^{-1} T^{\dagger} \xi\right\|^{2}=\left\|\delta T T^{\dagger} \xi\right\|=\|\delta \xi\|^{2}
$$

holds for all $\gamma$ and again it is impossible to reduce the nominal disturbance. If $N(T)^{\perp} \subset N(\delta T)$ then $K P=P K$. To verify this assertion note that $N(T)^{\perp} \subset$ $N(\delta T)$ implies $R(I-P) \subset N(\delta T)$ which implies $K(I-P)=0$. Taking adjoints shows that $(I-P) K=0$; hence by subtracting we have

$$
0=K(I-P)-(I-P) K=P K-K P
$$

which verifies the assertion. It was noted earlier that when $N(T)^{\perp} \subset N(\delta T)$ then $\delta \xi=\delta T T^{\dagger} \xi=0$ which cannot be improved upon.

\section{Example II.}

Another case relatively easy to analyze is where $T$ and $\delta T$ are functionals. Then

$$
P=I-\|T\|^{-2} T^{*} T \text { and }
$$

$$
\begin{aligned}
Q=\delta T P \delta T^{*} & =\delta T \delta T^{*}-\|T\|^{-2} \delta T T^{*} T \delta T^{*} \\
& =\|\delta T\|^{2}-\|T\|^{-2}|\langle T, \delta T\rangle|^{2} \\
& =\|\delta T\|^{2} \sin ^{2} \theta
\end{aligned}
$$

where $\theta$ is the angle between $T$ and $\delta T$ (i.e. $\cos \theta=\langle T, \delta T\rangle /\|T\| \cdot\|\delta T\|)$. Consequently,

$$
\begin{aligned}
f_{2}(\alpha) & =\alpha\left(1+\alpha\|\delta T\|^{2} \sin ^{2} \theta\right)^{-1}\|\delta \xi\|^{2} \\
f_{1}(\beta) & =\beta^{2}\left(1+\beta\|\delta T\|^{2} \sin ^{2} \theta\right)^{-2}\|\delta \xi\|^{2} \\
\left\|E_{\xi}\right\|^{2} & =\left(1+\left.\gamma\|\delta T\|\right|^{2} \sin ^{2} \theta\right)^{-2}\|\delta \xi\|^{2}
\end{aligned}
$$

Using these equations $\left\|E_{\xi}\right\|^{2}$ may be explicitly determined as a function of $\epsilon$. For instance, for Problem III the equation $f_{2}(\alpha)$. $=\epsilon\left\|T^{\dagger} \xi\right\|^{2}$ provides the relationships

$$
\begin{aligned}
\alpha & =\left(\|\delta \xi\|^{2}-\epsilon\left\|T^{\dagger} \xi\right\|^{2} \cdot\|\delta T\| \|^{2} \sin ^{2} \theta\right)^{-1} \epsilon\left\|T^{\dagger} \xi\right\|^{2} \\
\left(1+\alpha\|\delta T\|^{2} \sin ^{2} \theta\right) & =\alpha\|\delta \xi\|^{2} / \epsilon\left\|T^{\dagger} \xi\right\|^{2}
\end{aligned}
$$

from which it follows that

$$
\left(1+\alpha\|\delta T\|^{2} \sin ^{2} \theta\right)=\|\delta \xi\|^{2}\left(\|\delta \xi\|^{2}-\epsilon\left\|T^{\dagger} \xi\right\|^{2} \cdot\|\delta T\|^{2} \sin ^{2} \theta\right)^{-1}
$$

and consequently

$$
\left\|E_{\xi}\right\|=\left(\|\delta \xi\|^{2}-\epsilon\left\|T^{\dagger} \xi\right\|^{2} \cdot\|\delta T\|^{2} \sin ^{2} \theta\right) /\|\delta \xi\| .
$$


This equation, or the inequality

$$
\left\|E_{\xi}\right\| /\|\delta \xi\| \leq 1-\epsilon \sin ^{2} \theta
$$

(which follows easily from it) establishes a simple relationship between controller surplus capacity and the potential percentage reduction in disturbance.

\section{Example III.}

Consider the linear dynamic system satisfying the vector differential equation $\dot{x}(t)=A(t) x(t)+B(t) u(t), x(t)=x^{0}$. This system is to perform a fixed-time state transfer $\left(t_{0}, x^{0}\right) \rightarrow\left(t_{f}, x^{f}\right)$ with minimum energy. The transformation $T$ is identified by the equation

$$
T u=\int_{t_{0}}^{t_{s}} \Phi\left(t_{f}, s\right) B(s) u(s) d s
$$

while $\xi=x^{f}-\Phi\left(t_{f}, t_{0}\right) x^{0}$. Similarly $T^{*}$ and $T^{\dagger}$ can be directly computed. The matrix $B$ is assumed to depend on a parameter $\mu$ and by using a linearization this dependence can be expressed in the form

$$
B(t, \mu)=B_{1}(t)+\mu B_{2}(t)
$$

where $B_{1}$ and $B_{2}$ are fixed (known) matrices. The values $T$ and $\delta T$ are both defined as above with $B=B_{1}$ and $R=B_{2}$ in the two respective cases.

For any control $u$,

$$
\|\delta \xi\|^{2}=\mu^{2}\left\|\int_{t_{0}}^{t_{s}} \Phi\left(t_{f}, s\right) B_{2}(s) u(s) d s\right\|^{2} .
$$

holds. When $\mu$ is a random variable with zero mean $\delta \xi$ is a random vector with zero mean. The standard deviation $\sigma_{\mu}$ of $\mu$ and $\Sigma_{\xi^{2}}$ the expectation of $\|\delta \xi\|^{2}$, are related by

$$
\Sigma_{\xi}^{2}=\sigma_{\mu}^{2}\left\|\int_{t_{0}}^{t_{f}} \Phi\left(t_{f}, s\right) B_{2}(s) u(s) d s\right\|^{2}
$$

If the controller has surplus capacity it is natural to attempt a partial minimization of $\Sigma_{\xi \xi}$. It is obvious that such a problem falls within the framework of the present analysis. To complete the example the necessary computations are outlined.

If $u$ and $x$ are $m$ and $n$ tuples respectively the Hilbert spaces $H_{1}=\left[L_{2}\left(t_{0}, t_{f}\right)\right]^{m}$ and $H_{2}=E^{n}$ are the natural setting for the problem. It can be easily shown (see (9), sec. 3.3 ) that $T^{*}$ is computed by the equation

$$
\left(T^{*} \lambda\right)(t)=B_{1}^{*}(t) \Phi^{*}\left(t_{f}, t\right) \lambda, \quad t \in\left[t_{0}, t_{f}\right], \lambda \in E^{n}
$$


while $\delta T^{*}$ has the same form with $B_{2}$ replacing $B_{1}$. Since $P=I-T^{\dagger} T$ and assuming that the scalar $\gamma$ is small it follows that

$$
(I+\gamma P K)^{-1} T^{\dagger}=(I-\gamma P K) T^{\dagger}=T^{\dagger}-\gamma K T^{\dagger}+\gamma T^{\dagger} T K T^{\dagger} .
$$

Letting $N$ and $M$ denote the matrices

$$
\begin{aligned}
& N=T \delta T^{*}=\int_{t_{0}}^{t_{f}} \Phi\left(t_{f}, s\right) B_{1}(s) B_{2}^{*}(s) \Phi^{*}\left(t_{f}, s\right) d s \\
& M=T T^{*}=\int_{t_{0}}^{t_{s}} \Phi\left(t_{f}, s\right) B_{1}(s) B_{1}^{*}(s) \Phi^{*}\left(t_{f}, s\right) d s
\end{aligned}
$$

respectively (where $M$ is assumed to be nonsingular) the components of $(I+\gamma P K)^{-1} T^{\dagger}$ may be identified as

$$
\begin{aligned}
\left(T^{\dagger} \xi\right)(t) & =B_{1}^{*}(t) \Phi^{*}\left(t_{f}, t\right) M^{-1} \xi & & t \in\left[t_{0}, t_{f}\right] \\
\left(K T^{\dagger} \xi\right)(t) & =B_{2}^{*}(t) \Phi^{*}\left(t_{f}, t\right) N^{*} M^{-1} \xi & & t \in\left[t_{0}, t_{f}\right] \\
\left(T^{\dagger} T K T^{\dagger}\right)(t) & =B_{1}^{*}(t) \Phi^{*}\left(t_{f}, t\right) M^{-1} N N^{*} M^{-1} \xi & & t \in\left[t_{0}, t_{f}\right] .
\end{aligned}
$$

The specification of the open-loop controller is complete once $\gamma$ is determined. The only distinction here with the usual case is that $\sigma_{\mu}{ }^{2} \alpha$ (respectively $\sigma_{\mu}{ }^{2} \beta$ ) must be used in place of $\alpha$ (respectively $\beta$ ) in the determining equations.

Remark. When $x$ and $u$ are scalar valued functions Examples II and III may be combined (similar results hold for all single input angle output systems). Using the results of Example II it follows that

$$
\frac{\Sigma_{\xi}}{\|\delta \xi\| \sigma_{\mu}} \leq\left(1-\epsilon \sin ^{2} \theta\right)
$$

reflects the statistical improvement in the system performance.

\section{Discussion}

The fact that Problems I and II are equivalent may also be established as follows: For $\xi \in \mathrm{H}_{2}$ consider the solution to Problem II, namely

$$
T^{\# \xi}=(I-P) T^{\# \xi}+P T^{\# \xi}=u_{\xi}+v_{\xi}
$$

Obviously $u_{\xi} \in N(T)^{\perp}$ and $v_{\xi} \in N(T)$ and since $\xi=T T^{*} \xi$ it follows that $u_{\xi}=T^{\dagger} \xi$. It remains only to be shown that $v_{\xi}$ minimizes $\|\delta \xi+\delta T v\|$ over the set

$$
\left\{v \in N(T):\left\|v_{\xi}\right\|^{2}=\epsilon\left\|T^{\dagger} \xi\right\|^{2}\right\} \text {. }
$$

Assume that $v_{0} \neq v_{\xi}$ is the minimum element and that

$$
\left\|\delta \xi+\delta T v_{0}\right\|^{2}<\left\|\delta \xi+\delta T v_{\xi}\right\|^{2} .
$$


Since $v_{\xi}, v_{0} \in N(T)$ and have equal norms it is clear that

Moreover,

$$
\left\|u_{\xi}+v_{\xi}\right\|^{2}=\left\|u_{\xi}+v_{0}\right\|^{2} \text {. }
$$

$$
\begin{aligned}
J\left(u_{\xi}+v_{\xi}\right)= & \left\|u_{\xi}+v_{\xi}\right\|^{2}+\alpha\left\|\delta \xi+\delta T v_{\xi}\right\|^{2} \\
& >\left\|u_{\xi}+v_{0}\right\|^{2}+\alpha\left\|\delta \xi+\delta T v_{0}\right\|^{2}=J\left(u_{\xi}+v_{0}\right)
\end{aligned}
$$

which violates the definition of $T^{\#}$, namely, that $T^{\#} \xi$ minimizes $J$ over the set

$$
\left\{u \in T^{-1}(\xi):\|u\|^{2} \leq(1+\epsilon)\left\|T^{\dagger} \xi\right\|^{2}\right\}
$$

A similar contradiction may be constructed for establishing the equivalence in the opposite direction.

The comparison of Problems I and II with Problem III is a priori biased in favor of Problems I and II. Indeed, in the first two cases the energy constraint is of the form

while in the latter case

$$
\left\|T^{*} \xi\right\|^{2}=(1+\epsilon)\left\|T^{\dagger} \xi\right\|^{2}
$$

$$
\left\|T^{*} \xi\right\|^{2}+\alpha\left\|\delta T T^{*} \xi\right\|^{2}=(1+\epsilon)\left\|T^{\dagger} \xi\right\|^{2}
$$

must hold. Clearly, the second constraint is more restrictive than the first; hence the first case should be expected to result in a smaller system error. Thus these comparisons should be viewed as comparing the properties of the different constraints and not as a comparison of alternatives to the same problem.

Except in Example II when $T$ and $\delta T$ are functionals the functional dependence of $\alpha$ on $\epsilon$ is left in implicit form. Due to the monotonic behavior and the differentiability of the functions $f_{1}$ and $f_{2}$, it appears that the computational problem involved is only mildly difficult. For small $\epsilon, \alpha, \beta$ and $\gamma$ the first-order approximations

$$
\begin{aligned}
f_{2}(\alpha) & =\alpha\left\|\delta T T^{\dagger} \xi\right\|^{2}=\epsilon\left\|T^{\dagger} \xi\right\|^{2} \\
f_{1}(\beta) & =\beta^{2}\left\|P K T^{\dagger} \xi\right\|^{2}=\epsilon\left\|T^{\dagger} \xi\right\|^{2} \\
\left\|E_{\xi}\right\|^{2} & =\left\|\delta T T^{\dagger} \xi\right\|^{2}-2 \gamma\left\|P K T^{\dagger} \xi\right\|^{2}
\end{aligned}
$$

hold. Combining these equations, there results

$$
\left\|E_{\xi}\right\|^{2}=\|\delta \xi\|^{2}-2 \sqrt{\epsilon}\left\|T^{\dagger} \xi\right\| \cdot\left\|P K T^{\dagger} \xi\right\|
$$

for Problems I and II, and

$$
\left\|E_{\xi}\right\|^{2}=\|\delta \xi\|^{2}-2 \epsilon\left\|T^{\dagger} \xi\right\|^{2}
$$

for Problem III. These two system error equations show an obvious difference in the dependence between $\left\|E_{\xi}\right\|^{2}$ and $\epsilon$. 


\section{Appendix}

In the following, $H$ denotes an abstract Banach space. The principal tool to be used in this Appendix is supplied by the following theorem (see (11), p. 164).

Theorem. Let $A$ and $S$ be bounded linear operators on $H$. If $\|A\|<1$ then $I-A$ has a bounded inverse and

$$
(I-A)^{-1}=I+A+A^{2}+A^{3}+\cdots
$$

If $S$ commutes with $A$ and if $\left\|(I-A)^{-1} S\right\|<1$ then $I-S-A$ has a bounded inverse and

$$
(I-S-A)^{-1}=(I-A)^{-1}\left\{I+(I-A)^{-1} S+(I-A)^{-2} S^{2}+\cdots\right\}
$$

Both series converging with respect to the operator norm.

Corollary $I$. Let $Q$ be a self adjoint operator on $H$ then the functions

$$
\begin{aligned}
& f_{1}(\gamma)=\gamma^{2}\left\langle\eta, Q(I+\gamma Q)^{-2} \eta\right\rangle \\
& f_{2}(\gamma)=\gamma\left\langle\eta,(I+\gamma Q)^{-1} \eta\right\rangle
\end{aligned}
$$

are monotone nondecreasing with respect to $\gamma$.

Proof: Let $\delta$ be a small scalar. Then letting $A=-\gamma Q$ and $S=-\delta Q$ the above theorem provides the identity

$$
\begin{aligned}
{[I+(\gamma+\delta) Q]^{-1}=} & (I+\gamma Q)^{-1} \\
& \times\left\{I-\delta(I+\gamma Q)^{-1} Q+\delta^{2}(I+\gamma Q)^{-2} Q^{2}-\cdots\right\} .
\end{aligned}
$$

from which it follows that

$$
\left\langle\eta,[I+(\gamma+\delta) Q]^{-1} \eta\right\rangle=\left\langle\eta,(I+\gamma Q)^{-1} \eta\right\rangle-\delta\left\langle\eta, Q(I+\gamma Q)^{-2} \eta\right\rangle+I\left(\delta^{2}\right)
$$

and therefore

$$
\frac{d}{d \gamma}\left\langle\eta,(I+\gamma Q)^{-1} \eta\right\rangle=-\left\langle\eta, Q(I+\gamma Q)^{-2} \eta\right\rangle
$$

It then follows that

$$
\frac{d}{d \gamma} f_{2}(\gamma)=\left\langle\eta,(I+\gamma Q)^{-1} \eta\right\rangle-\gamma\left\langle\eta, Q,(I+\gamma Q)^{-2} \eta\right\rangle
$$

Using the identity $(I+\gamma Q)^{-1}-\gamma Q(I+\gamma Q)^{-2}=(I+\gamma Q)^{-2}$ it follows easily 
that

$$
\frac{d}{d \gamma} f_{2}(\gamma)=\left\langle\eta,(I+\gamma Q)^{-2} \eta\right\rangle \geq I
$$

Since term-wise multiplication of two absolutely convergent series is permissible, the identity

$$
\begin{aligned}
{[I+(\gamma+\delta) Q]^{-2} } & =(I+\gamma Q)^{-2} \\
\times & \left\{I-2 \delta(I+\gamma Q)^{-1} Q+3 \delta^{2}(I+\gamma Q)^{-2} Q^{2}-\cdots\right\}
\end{aligned}
$$

follows from Eq. A.1. As a consequence

$$
\frac{d}{d \gamma}\left\langle\eta, Q(I+\gamma Q)^{-2} \eta\right\rangle=-2\left\langle\eta, Q^{2}(I+\gamma Q)^{-3} \eta\right\rangle
$$

holds; hence

$$
\begin{aligned}
\frac{d}{d \gamma} f_{1}(\gamma) & =2 \gamma\left\langle\eta, Q(I+\gamma Q)^{-2} \eta\right\rangle-2 \gamma^{2}\left\langle\eta, Q^{2}(I+\gamma Q)^{-3} \eta\right\rangle \\
& =2 \gamma\left\langle\eta, Q(I+\gamma Q)^{-3} \eta\right\rangle \geq 0
\end{aligned}
$$

which completes the proof.

Corollary II. If $Q$ is a self-adjoint operator on $H$ then

$$
f_{3}(\gamma)=\left\langle\eta,(I+\gamma Q)^{-2} \eta\right\rangle
$$

is a nonincreasing function of $\gamma$. Moreover,

$$
f_{4}(\gamma)=f_{2}(\gamma)-f_{1}(\gamma)
$$

is a nonnegative function of $\gamma$.

Proof: Using Eq. A.2 it follows that

$$
\frac{d}{d \gamma} f_{3}(\gamma)=-2\left\langle\eta, Q(I+\gamma Q)^{-3} \eta\right\rangle \leq 0
$$

which proves the first assertion. From the identity

it follows that

$$
\gamma(I+\gamma Q)^{-1}-\gamma^{2} Q(I+\gamma Q)^{-2}=\gamma(I+\gamma Q)^{-2}
$$

$$
f_{4}(\gamma)=\gamma\left\langle\eta,(I+\gamma Q)^{-2} \eta\right\rangle \geq 0
$$

which completes the proof.

Note from the proofs of Corollaries I and II that if $Q$ is strictly positive then 
$f_{1}$ and $f_{2}$ are strictly monotonically increasing, $f_{3}$ is strictly monotonically decreasing, and $f_{4}$ is strictly positive on the domain $0<\gamma<\infty$.

This research was supported by the U. S. Army Research Office-Durham under Contract DA-31-124 ARD-D-391 and by the U. S. Air Force under contract AF-30(602)-3546.

\section{References}

(1) B. Pagurek, "Sensitivity of the Performance of Optimal Control Systems to Plant Parameter Variations," IEEE Trans. Auto. Control Vol. AC-10, pp. 178-180, Apr. 1965.

(2) H. S. Witsenhausen, "On the Sensitivity of Optimal Control Systems," IEEE' Trans. Auto. Control (Corresp.) Vol. AC-10, No. 4, pp. 495-496, Oct. 1965.

(3) J. M. Holtzman and S. Horing, "The Sensitivity of Terminal Conditions of Optimal Control Systems to Parameter Variations," IEEE Trans. Auto. Control, Vol. AC-10, No. 4, pp. 420-426, Oct. 1965.

(4) W. A. Porter, "On Singularities that Arise in a Class of Optimal Feedback Systems," IEEE Trans. Auto. Control, Vol. AC-11, No. 4, pp. 617-618, July 1966.

(5) R. A. Rohrer and M. Sobral, "Sensitivity Considerations in Optimal System Design," IEEE Trans. Auto. Control, Vol. AC-10, No. 1, pp. 43-48, Jan. 1965.

(6) W. A. Porter, "Sensitivity Problems in Linear Systems," IEEE Trans. Auto. Control, Vol. AC-10, No. 3, pp. 301-307, July 1965.

(7) W. R. Perkings and J. B. Cruz, Jr., "The Parameter Variation Problem in State Feedback Control Systems," presented at the Joint Auto. Control Conf., Stanford University. Stanford, Calif., 1964.

(8) W. A. Porter, "Some Theoretical Limitations of System Sensitivity Reduction," Proc. Third Annual Allerton Conference on Circuit and System Theory, pp. 241-251, Univ, of Illinois, Urbana, Oct. 1965.

(9) W. A. Porter, "Modern Foundations of System Analysis," New York, The Macmillan Co., 1966.

(10) W. A. Porter, "Concerning Disturbance Measures in Linear Systems," IEEE Trans. Auto. Control, Vol. AC-11, No. 3, pp. 532-534, July 1966.

(11) A. E. Taylor, "Introduction to Functional Analysis," New York, John Wiley and Sons, 1961. 